\title{
Analyzing the Transitional Region in Low Power Wireless Links
}

\author{
Marco Zuniga and Bhaskar Krishnamachari \\ Department of Electrical Engineering - Systems \\ University of Southern California, Los Angeles, CA 90089-0781 \\ marcozun@usc.edu,bkrishna@usc.edu
}

\begin{abstract}
The wireless sensor networks community, has now an increased understanding of the need for realistic link layer models. Recent experimental studies have shown that real deployments have a "transitional region" with highly unreliable links, and that therefore the idealized perfect-reception-within-range models used in common network simulation tools can be very misleading. In this paper, we use mathematical techniques from communication theory to model and analyze low power wireless links. The primary contribution of this work is the identification of the causes of the transitional region, and a quantification of their influence. Specifically, we derive expressions for the packet reception rate as a function of distance, and for the width of the transitional region. These expressions incorporate important channel and radio parameters such as the path loss exponent and shadowing variance of the channel; and the modulation and encoding of the radio. A key finding is that for radios using narrow-band modulation, the transitional region is not an artifact of the radio non-ideality, as it would exist even with perfectthreshold receivers because of multi-path fading. However, we hypothesize that radios with mechanisms to combat multi-path effects, such as spread-spectrum and diversity techniques, can reduce the transitional region.
\end{abstract}

\section{INTRODUCTION}

Wireless sensor network protocols are often evaluated through simulations that make simplifying assumptions about the link layer, such as the binary perfect-reception-withinrange model. Several recent empirical studies [1] [2] [3] have questioned the validity of these assumptions. These studies have revealed the existence of three distinct reception regions in a wireless link: connected, transitional, and disconnected. The transitional region is often quite significant in size, and is generally characterized by high-variance in reception rates and asymmetric connectivity. Particularly, in dense deployments such as those envisioned for sensor networks, a large number of the links in the network (even higher than 50\%) can be unreliable due to the transitional region.

Because of its inherent unreliability and extent, the transitional region can have a major impact on the performance of upper-layer protocols. In [1] it is shown that the dynamics of even the simplest flooding mechanism and the topology of data gathering trees constructed in dense sensor networks can be significantly affected due to the asymmetric and occasional long-distance links caused by nodes present in the transitional region. In [6] also, it is argued that the

This work was supported in part by NSF under grant number 0347621, and by a gift grant from Ember Corporation. routing structures formed taking into account unreliable links can be very different from the structures formed based on a simplistic model. Similarly, the authors of [7] report that such unreliable links can have a significant impact on routing protocols, particularly geographic forwarding schemes. On the other hand, other works have proposed mechanisms to take advantage of nodes in the transitional region. For instance, [5] found that protocols using the traditional minimum hopcount metric perform poorly in terms of throughput, and that a new metric called ETX (expected number of transmissions), which uses nodes in the transitional region, has the best performance. On the same line of work, by evaluating link estimator and neighborhood table management, the authors in [3] found that cost-based routing using a minimum expected transmission metric has a good performance. Therefore, due to the significant impact that nodes in the transitional region have on upper-layer protocols, there is an increased understanding of the need for realistic link layer models for wireless sensor networks.

In order to address this need, some recent works [3] [7] [8] have proposed new link models based on empirical data. While these empirical models do play an invaluable role in improving the realism of protocol evaluation, they suffer from some significant shortcomings. They do not provide fundamental insight into the root causes of the observed phenomena. And they do not provide a systematic way to generalize the models (i.e., extend their validity and accuracy) beyond the specific radio and environment conditions of the experiments from which the models are derived.

On the other hand, there exists a rich literature on wireless communications, particularly in the context of cellular telecommunication networks ${ }^{1}$, that provides a set of models and tools for analyzing the physical layer. In this study, we make use of these analytical tools to derive expressions for the packet reception rate as a function of distance for different settings, and to determine the width of the transitional region. These expressions do not consider node mobility nor dynamic objects in the environment; thus, while different links experience different levels of fading, the fading for each link is assumed to be constant over time.

The analysis done in this work provides some important contributions. First, it allows us to delimit the influence of

\footnotetext{
${ }^{1}$ In cellular systems the transitional region is not of interest (except for modelling inter-cell interference) as cells are designed to fit only the connected region.
} 
the wireless environment and the radio on the transitional region; furthermore, the derived expressions show how the transitional region is impacted by important radio parameters such as modulation, encoding, output power, frame size and receiver noise, as well as important environmental parameters, namely, the path loss exponent and the log-normal shadow variance. Second, we are able to conclude that, for radios using narrow-band modulation, the transitional region is present even with perfect-threshold radios (i.e., that it is not an artifact of radio non-ideality alone) due to shadowing effects; hence, radios with mechanisms to combat multi-path may reduce the transitional region. And third, we bring to the notice of the community simple analytical models for the link layer that can be used to enhance simulations.

The rest of the paper is organized as follows. Section II positions our work in the current literature. The basic framework of the model is derived in section III, it shows how the channel and radio influence the transitional region. In section IV, the model is extended for different environments, encoding schemes, and frames size. This section also introduces the transitional region coefficient $\Gamma$ as a mean to measure the quality of the link by taking into account the width of the different regions. Section V shows empirical experiments used to validate and enhance the correctness of the model, and provides theoretical models for several scenarios. Finally, we present our conclusions and future work in section VI.

\section{RELATED WORK}

Recent experimental studies [1] [2] [3] identify the existence of three distinct reception regions in the wireless link: connected, transitional, and disconnected. This behavior deviate to a large extend from the idealized disc-shape model used in most published results. In [6], Kotz et al. provide data demonstrating the unrealistic nature of some common assumptions used in MANET research. In real scenarios, packet losses lead to different connectivity graphs, and coverage ranges that are neither circular nor convex, and are often noncontiguous.

Several researchers have pointed out that the use of simple radio models may lead to wrong simulation results in upperlayers. In one of the earliest works, Ganesan et al. [1] presented empirical results from flooding in a dense sensor network and study different effects at the link, MAC, and application layers. They found that the flooding tree exhibits a high clustering behavior, in contrast to the more uniformly distributed tree obtained with a disc shape model.

Zhao et al. [2] report measurements of packet delivery for a sixty-node test-bed in different indoor and outdoor environments. They study the impact of the wireless link in packet delivery at the physical and MAC layers by testing different encoding schemes (physical layer) and different traffic loads (MAC layer).

In [5], De Couto et al. present measurements for DSDV and DSR, over a 29 node 802.11 b test-bed and show that when the real channel characteristics are not taken into account, the minimum hop-count metric has poor performance. By incorporating the effects of link loss ratios, asymmetry, and interference, they present the expected transmission count metric which finds high throughput paths. On the same line of work, Woo et al. [3] study the effect of link connectivity on distance-vector based routing in sensor networks. By evaluating link estimator, neighborhood table management, and reliable routing protocols techniques, they found that costbased routing using a minimum expected transmission metric shows good performance.

Recently, Zhou et al. [7] reported that radio irregularity has a significant impact on routing protocols, but a relatively small impact on MAC protocols. They found that location-based routing protocols, such as geographic routing perform worse in the presence of radio irregularity than on-demand protocols, such as AODV and DSR.

Through empirical studies, the previous works bring to light the impact that the channel behavior has on protocol performance at different layers. However, for large-scale networks, on-site testing may be unfeasible and models for simulators will be needed. In order to help overcoming this problem some tools and models have been recently proposed.

In [3], the authors derive a packet loss model based on aggregate statistical measures such as mean and standard deviation of packet reception rate (PRR). The model assumes a gaussian distribution of the PRR for given transmitter-receiver distance, which is not accurate.

Using the SCALE tool [4], Cerpa et al. [8] identify other factors for link modelling. They capture features of groups of links associated with a particular receiver, a particular transmitter, a particular radio, and links associated with a group of radios that are geographically close. Using several statistical techniques, they provide a spectrum of models of increasing complexity and increasing accuracy.

A most recent model, called the Radio Irregularity Model (RIM), was proposed in [7]. Based on experimental data, RIM takes into account both the non-isotropic properties of the propagation media and the heterogeneous properties of devices.

While these models are important steps towards a realistic channel model, their main drawback is that they are valid only for the parameters used in the deployment; among those we have: modulation, encoding, packet size, environment characteristics, noise floor and output power. If these parameters are modified the empirical model is either not valid or not accurate.

On the other hand, years of research in wireless communications, particularly cellular networks, provide a rich set of models and tools for analyzing the physical layer [13]. Two of these tools are of significant importance to understand the transitional region, the log-normal shadowing path loss model (to model the environment) and the bit-error performance of various modulation and encoding schemes with respect to the signal to noise ratio (to model the radio).

The research done so far has identified the channel modelling problem and its impact on upper-layer protocols, it also has proposed some realistic channel models. However, what is missing is a clear understanding of the causes of the link behavior. Our work presents an in-depth analysis of the transitional region and provides theoretical models for the link layer showing how PRRs vary with distance for 


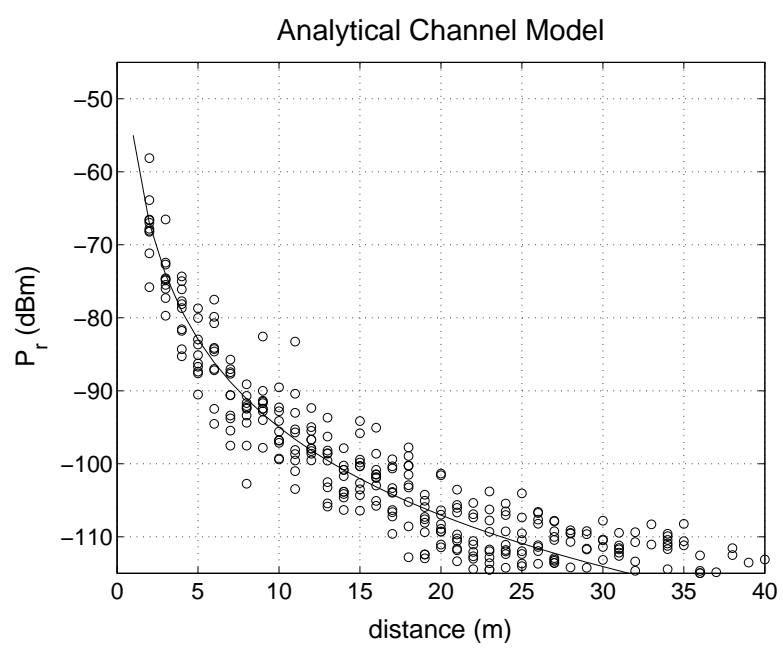

Fig. 1. Channel Model, $n=4, \sigma=4, P_{t}=0 \mathrm{dBm}$

different radios and environments. The model presented in this work does not consider interference, which is part of our future work. Nevertheless, in scenarios where the traffic and contention are relatively light; a very reasonable assumption for many classes of data-centric sensor networks, our model provides an accurate estimate of the links' quality.

\section{Delimiting Responsibilities: The Channel And THE RADIO}

The transitional region is the result of placing specific devices, for example MICA2 motes, in an specific environment, like the aisle of a building. With the intend of analyzing how the channel and the radio determine the transitional region; first, we define models for both elements, to subsequently study their interaction.

\section{A. The Wireless Channel}

When an electromagnetic signal propagates, it may be diffracted, reflected and scattered. These effects have two important consequences on the signal strength. First, the signal strength decays exponentially with respect to distance. And second, for a given distance $d$, the signal strength is random and log-normally distributed about the mean distancedependent value.

Due to the unique characteristics of each environment, most radio propagation models use a combination of analytical and empirical methods. One of the most common radio propagation models is the log-normal shadowing path loss model $[13]^{2}$. This model can be used for large and small [11] coverage systems; furthermore, empirical studies [12] have shown the the log-normal shadowing model provides more accurate multi-path channel models than Nakagami and Rayleigh for indoor environments. The model is given by:

$$
P L(d)=P L\left(d_{0}\right)+10 n \log _{10}\left(\frac{d}{d_{0}}\right)+X_{\sigma}
$$

\footnotetext{
${ }^{2}$ The model is valid only for the transmission frequency and environment where the data was gathered.
}

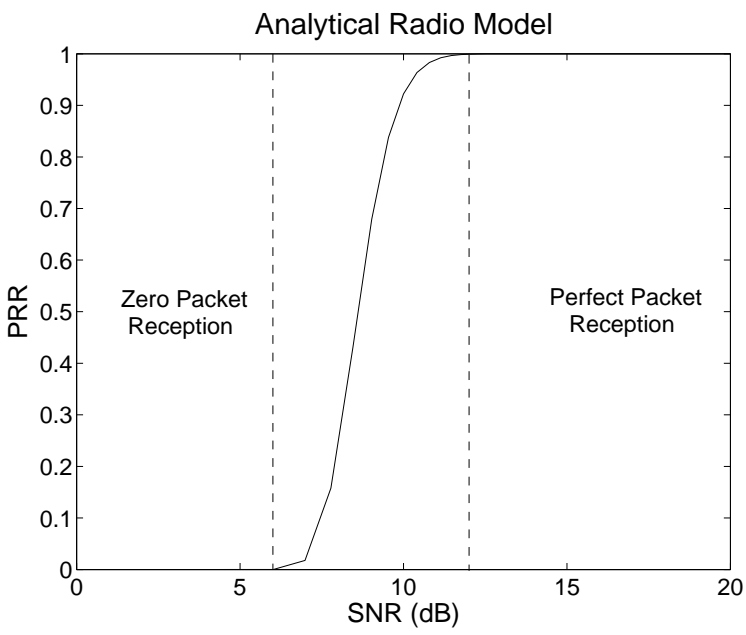

Fig. 2. Radio Model: Non-Coherent FSK, NRZ radio, $f=50$ bytes

Where $d$ is the transmitter-receiver distance, $d_{0}$ a reference distance, $n$ the path loss exponent (rate at which signal decays), and $X_{\sigma}$ a zero-mean Gaussian RV (in dB) with standard deviation $\sigma$ (shadowing effects) ${ }^{3}$. In the most general case, $X_{\sigma}$ is a random process that is a function of time, but, since we are not assuming dynamic environments, we model it as a constant random variable over time for a particular link.

The received signal strength $\left(P_{r}\right)$ at a distance $d$ is the output power of the transmitter minus $P L(d)$. Figure 1 shows an analytical propagation model for $n=4, \sigma=4, P L\left(d_{0}\right)=$ $55 \mathrm{~dB}$ and an output power of $0 \mathrm{dBm}$.

\section{B. The Radio}

To facilitate the explanation of the radio model, this subsection assumes NRZ encoding. Section IV provides models for other encoding schemes.

The steps followed to derive the radio model are similar to the ones in [9]. Let $P_{i}$ be a Bernoulli random variable, where $P_{i}$ is 1 if the packet is received and 0 otherwise. Then, for $r$ transmissions, the packet reception rate is defined by $\frac{1}{r} \sum_{i=1}^{r} P_{i}$. Since $P_{i}$ s are i.i.d. random variables, by the weak law of large numbers PRR can be approximated by $E\left[P_{i}\right]$, where $E\left[P_{i}\right]$ is the probability of successfully receiving a packet.

If NRZ is used and 1 Baud $=1$ bit, the probability $p$ of successfully receiving a packet is:

$$
\begin{aligned}
p & =\left(1-P_{e}\right)^{8 \ell}\left(1-P_{e}\right)^{8(f-\ell)} \\
& =\left(1-P_{e}\right)^{8 f}
\end{aligned}
$$

Where $f$ is the frame size ${ }^{4}, \ell$ is the preamble (both in bytes), and $P_{e}$ is the probability of bit error. $P_{e}$ depends on the modulation scheme, for non-coherent FSK (modulation used in MICA2 motes), $P_{e}$ is given by:

$$
P_{e}=\frac{1}{2} \exp ^{-\frac{\alpha}{2}}
$$

\footnotetext{
${ }^{3} n$ and $\sigma$ are obtained through curve fitting of empirical data; $P L\left(d_{0}\right)$ can be obtained empirically or analytically.

${ }^{4} \mathrm{~A}$ frame consists of: preamble, network payload (packet) and CRC
} 
Analytical Method to Determine Regions in Wireless Links

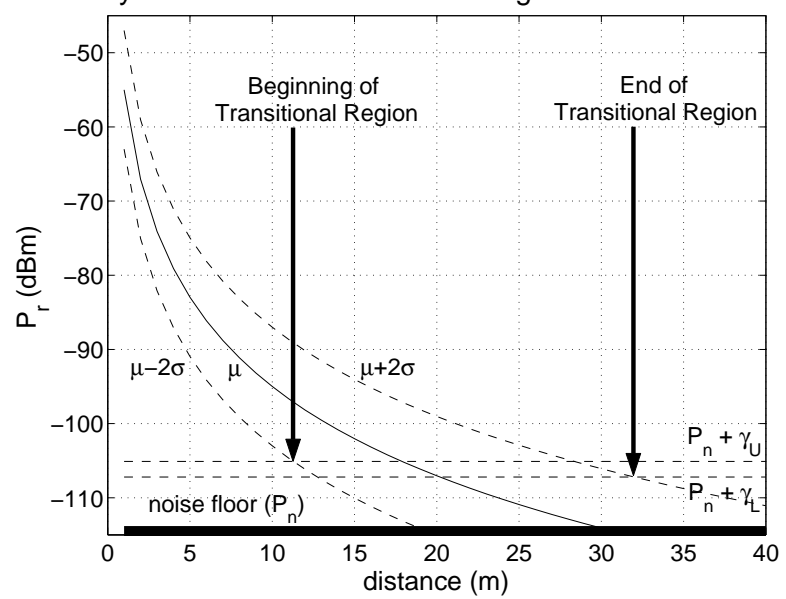

Fig. 3. Analytical Observation of the Transitional Region

Where $\alpha$ is the $\frac{E_{b}}{N_{0}}$ ratio. Hence, the PRR $p$ is defined as:

$$
p=\left(1-\frac{1}{2} \exp ^{-\frac{\alpha}{2}}\right)^{8 f}
$$

Nevertheless, most commercial radios do not provide the $\frac{E_{b}}{N_{0}}$ metric, but the RSSI (Received Signal Strength Indicator) of the received signal. The RSSI measurements can be used to determine the SNR (Signal-to-Noise ratio); henceforth, in this work, the expression based on $\frac{E_{b}}{N_{0}}$ are converted to SNR.

The relation between SNR and $\frac{E_{b}}{N_{0}}$ is given by:

$$
S N R=\frac{E_{b}}{N_{0}} \frac{R}{B_{N}}
$$

Where $R$ is the data rate in bits, and $B_{N}$ is the noise bandwidth. For MICA2 motes, $R=19.2 \mathrm{kbps}$ and $B_{N}=30$ $\mathrm{kHz}$. Finally, the PRR $p$ in terms of the SNR $(\gamma)$ is given by:

$$
p=\left(1-\frac{1}{2} \exp ^{-\frac{\gamma}{2} \frac{1}{0.64}}\right)^{8 f}
$$

The curve in figure 2 shows equation 6 (receiver response) for a frame size of 50 bytes. As we shall see later, this curve plays an important role in determining the different regions.

\section{The Noise Floor}

Another important element that determines the transitional region is the noise floor, which depends on both, the radio and the environment. The temperature of the environment influences the thermal noise generated by the radio components (noise figure), the environment can further influence the noise floor due to interfering signals. When the receiver and the antenna have the same ambient temperature the noise floor is given by [13]:

$$
P_{n}=(F+1) k T_{0} B
$$

Where $F$ is the noise figure, $k$ the Boltzmann's constant, $T_{0}$ the ambient temperature and $B$ the equivalent bandwidth. MICA2s use the Chipcon CC1000 radio [14], which has a noise figure of $13 \mathrm{~dB}$ and a system noise bandwidth of 30

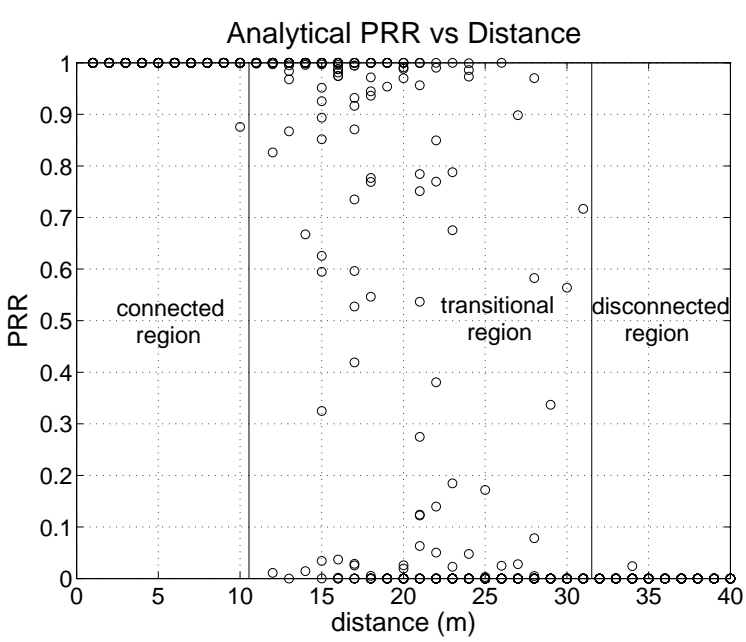

Fig. 4. Analytical PRR vs Distance, obtained through equation 9

$\mathrm{kHz}$. Considering an ambient temperature of $300{ }^{\circ} \mathrm{K}\left(27{ }^{\circ} \mathrm{C}\right.$, $75^{\circ} \mathrm{F}$ ) and no interference signals, the noise floor is $-115 \mathrm{dBm}$.

The noise figure provided in [14] is only for the chip, and does not include losses due to board implementations. Hence, the noise figure of the final hardware will be higher. In section $\mathrm{V}$, the noise floor is redefined based on empirical measurements.

\section{Putting all Together}

Given a transmitting power $P_{t}$, the SNR $\gamma$ at a distance $d$ is:

$$
\gamma(d)_{d B}=P_{t d B}-P L(d)_{d B}-P_{n d B}
$$

Henceforth, the PRR at a distance $d$ for the encoding and modulation assumed in this section is:

$$
p(d)=\left(1-\frac{1}{2} \exp ^{-\frac{\gamma(d)}{2} \frac{1}{0.64}}\right)^{8 f}
$$

With the aim of obtaining the radius of the different regions, let us bound the connected region to PRRs greater than 0.9, and the transitional region to values between 0.9 and 0.1 . If we let $\gamma_{U d B}$ and $\gamma_{L d B}$ be the SNR values for PRRs of 0.9 and 0.1 respectively, then from equation 9 we obtain:

$$
\begin{aligned}
& \gamma_{U d B}=10 \log _{10}\left(-1.28 \ln \left(2\left(1-0.9^{\frac{1}{8 f}}\right)\right)\right) \\
& \gamma_{L d B}=10 \log _{10}\left(-1.28 \ln \left(2\left(1-0.1^{\frac{1}{8 f}}\right)\right)\right)
\end{aligned}
$$

The previous equations determine the bounds of the regions in the radio model. Now, let us analyze how these bounds interact with the channel model to define the radius of the different regions at the link layer.

Due to the gaussian characteristic of log-normal shadowing in the path loss model, the received signal strength $P_{r}$ can be bounded within $\pm 2 \sigma$, i.e. $P\left(\mu-2 \sigma<P_{r}<\mu+2 \sigma\right)=.955$. If we let $\overline{P L}(d)=P L\left(d_{0}\right)+10 n \log _{10}\left(\frac{d}{d_{0}}\right)$, then, for a given output power $P_{t}$, the received power $P_{r}$ at a distance $d$ is bounded by: 


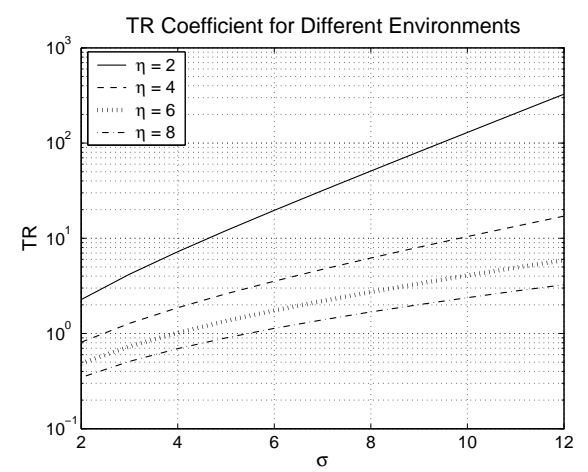

(a)

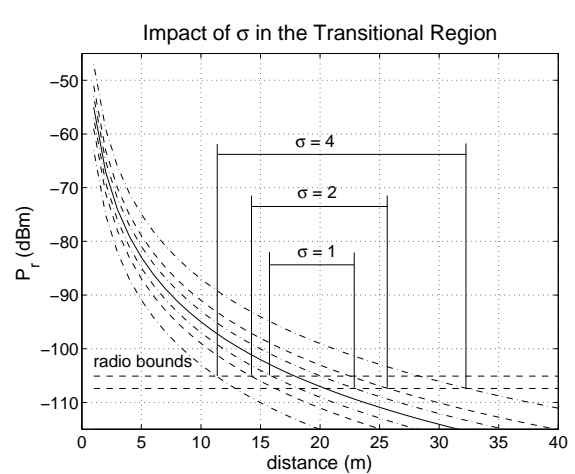

(b)

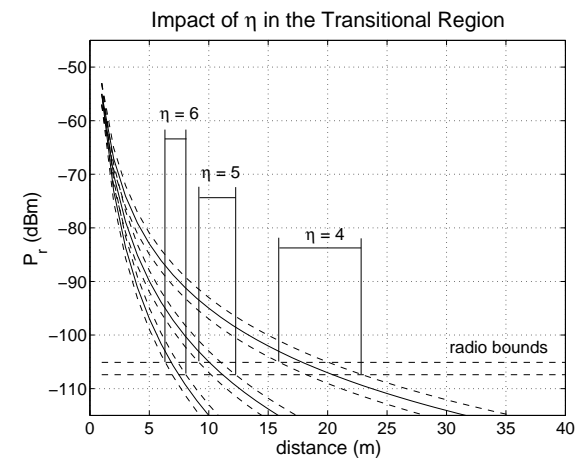

(c)

Fig. 5. Impact of the path loss exponent $n$ and the log-normal shadowing standard deviation $\sigma$ on the Transitional Region. (a) $\Gamma$ coefficient for various $\sigma$ and $n$. (b) if $\sigma$ increases, the transitional region increases. (c) if $n$ increases, the transitional region decreases

$$
\begin{aligned}
& P_{r U}(d)=P_{t}-\overline{P L}(d)+2 \sigma \\
& P_{r L}(d)=P_{t}-\overline{P L}(d)-2 \sigma
\end{aligned}
$$

Figure 3 shows the physical interaction of the channel and radio models (equations 10 and 11). The transitional region begins when the $P_{r}$ values $\left(P_{r L}\right)$ enter the $P_{n}+\gamma_{U}$ limit, and ends when the $P_{r}$ values $\left(P_{r U}\right)$ leave the $P_{n}+\gamma_{L}$ limit. By combining equations 10 and 11 , we obtain the conditions for the limits of the different regions:

$$
\begin{aligned}
& P_{r U}=\gamma_{L}+P_{n} \\
& P_{r L}=\gamma_{U}+P_{n}
\end{aligned}
$$

Finally, the beginning $\left(d_{s}\right)$ and end $\left(d_{e}\right)$ of the transitional region are given by:

$$
\begin{aligned}
& d_{s}=10^{\frac{P_{n}+\gamma_{U}-P_{t}+P L\left(d_{0}\right)+2 \sigma}{-10 n}} \\
& d_{e}=10^{\frac{P_{n}+\gamma_{L}-P_{t}+P L\left(d_{0}\right)-2 \sigma}{-10 n}}
\end{aligned}
$$

Equation 13 provides absolute values for the radius of the regions. However, a comparison of the regions' size between different scenarios may be desirable. With that aim, we define the transitional region coefficient $\Gamma$, which is the ratio of the radius of the transitional and connected regions. This coefficient is defined as:

$$
\Gamma=\frac{d_{e}-d_{s}}{d_{s}}
$$

Which leads to:

$$
\Gamma=10^{\frac{\left(\gamma_{U}-\gamma_{L}\right)+4 \sigma}{10 n}}-1
$$

The lower the coefficient the better, since that implies a larger connected region compared to the transitional one. For example, in the disc-shape model, $\sigma=0$ and $\gamma_{U}=\gamma_{L}$, which leads to $\Gamma=0$. It is interesting to observe that the ratio between the regions is independent from the noise floor and output power.

For the parameters chosen in this section (frame size $f=$ 50 bytes and transmitting power $P_{t}=0 \mathrm{dBm}$ ), we obtain $\gamma_{U}$ $=9.9 \mathrm{~dB}$ and $\gamma_{L}=7.6 \mathrm{~dB}$, which leads to $d_{s}=11.3 \mathrm{~m}, d_{e}$ $=32.4 \mathrm{~m}$ and $\Gamma=1.9$. Figure 4 shows the analytical PRR vs distance obtained by equation 9 , we observe that the beginning and end of the transitional region match the analytical values obtained.

\section{Detailed Analysis Of The Model}

The theoretical model derived in the previous section provides a general framework to evaluate the transitional region. In this section, we extend the model for different environments and radio characteristics.

Due to space constraints, we focus the analysis of the model on non-coherent FSK, which is the modulation used in the MICA2 architecture. This modulation technique will be the basis to compare the $\Gamma$ coefficients of diverse environments, with different encoding schemes and frames size. The probability of bit error for other modulation techniques are widely available [13], and can be easily inserted in the model. Section V provides expressions for some common modulation techniques.

\section{A. Fix the Radio Characteristics, Modify the Environment}

Due to the numerous applications envisioned for wireless sensor networks, an specific device will be required to work in different environments. The model allows to estimate the influence that the environment has in the different regions, which can be used to evaluate the performance of the network.

Usually a wireless channel is considered benign if both, the path loss exponent $n$ and the shadowing standard deviation $\sigma$ are small. Nevertheless, as figure 5 (a) shows, while a small $\sigma$ decreases the $\Gamma$ coefficient, a small $n$ increases it. Henceforth, scenarios with high $n$ and low $\sigma$ are preferable in terms of the $\Gamma$ coefficient. Figures 5 (b) and (c) show the physical impact of these two parameters in the transitional region.

In figure 5 (b), $n$ is set to 4 and $\sigma$ has values of 1,2 and 4 . The SNR bounds of the radio model are fixed and independent of the environment, hence, as $\sigma$ increases, the $P_{r}$ values have a higher probability of entering the transitional region at closer distances from the transmitter, and leaving it at farther distances; which results in a larger transitional region.

Figure 5 (c) shows the impact of $n$; $\sigma$ is set to 1 and $n$ takes values of 4,5 and 6 ; the higher $n$, the faster the decay of the 


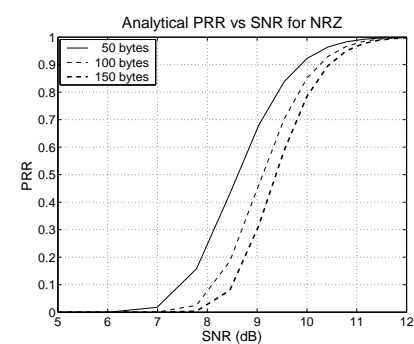

(a)

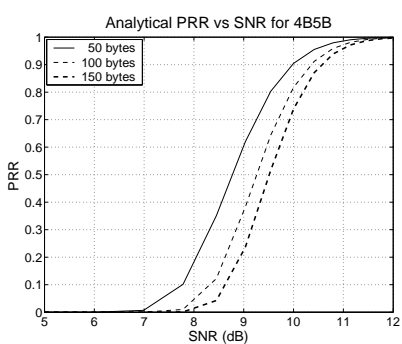

(b)

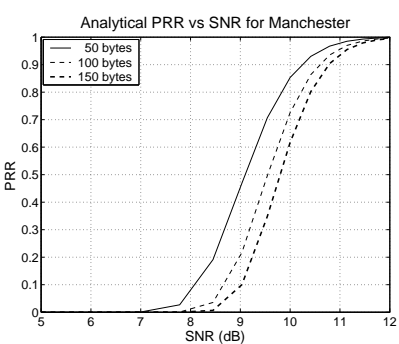

(c)

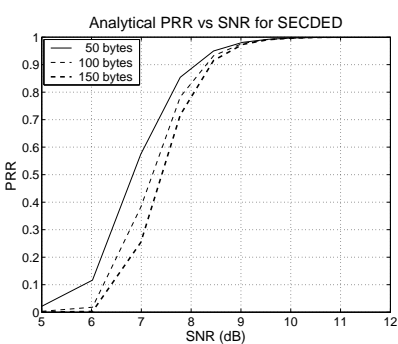

$(d)$

Fig. 6. Receiver Response for Different Encoding Schemes and Frames Size: (a) NRZ, (b) 4B5B, (c) Manchester, (d) SECDED

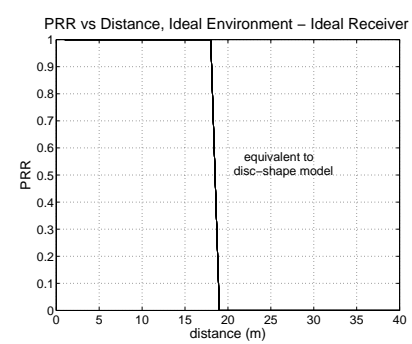

(a)

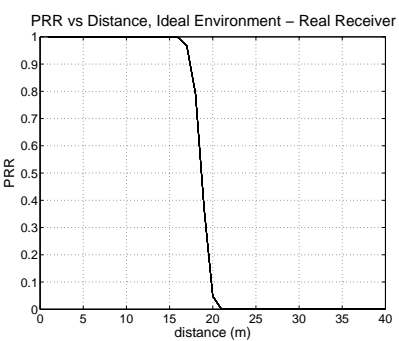

(b)

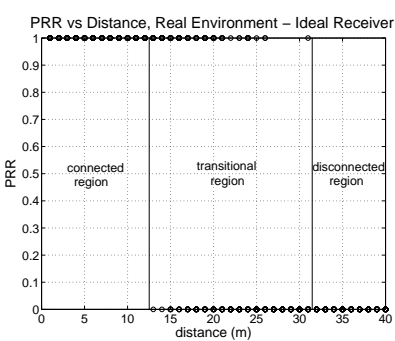

(c)

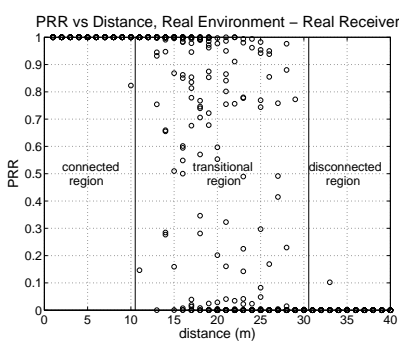

(d)

Fig. 7. PRR vs Distance for Ideal and Real Scenarios.

signal strength and the thinner the width of the transitional region.

Finally, it is important to mention that even though figure 5 (a) shows the curves of a radio using NRZ and frames of 50 bytes, similar trends are observed for other encoding schemes and frames size.

\section{B. Fix the Environment, Modify the Radio Characteristics}

Some WSN applications will require the optimization of the radio for a specific environment. In these cases, it will be important to explore different encoding schemes and observe the influence of the frame size.

Figure 6 shows the radio model (PRR vs SNR) for different encoding schemes ${ }^{5}$ (NRZ, 4B5B, Manchester and SECDED ${ }^{6}$ ), and various frames size.

For any encoding scheme, as the frame size increases, the SNR bounds increase (curves shift right) which leads to smaller connected regions. On the other hand, for the same frame size, the SNR bounds required by SECDED are the smallest (largest connected region), followed by NRZ, 4B:5B and Manchester. This result is due to the error correction

\footnotetext{
${ }^{5}$ The plots represent frames with a preamble length of 2 bytes for all encoding schemes.

${ }^{6}$ SECDED encodes each byte into 24 bits
}

\begin{tabular}{|c|c|c|c|}
\hline encoding & 50 bytes & 100 bytes & 150 bytes \\
\hline NRZ & 2.2785 & 2.0347 & 1.9151 \\
4B5B & 2.1938 & 1.9671 & 1.8551 \\
Manchester & 2.0347 & 1.8384 & 1.7404 \\
SECDED & 2.5677 & 2.2180 & 2.0489 \\
\hline
\end{tabular}

TABLE I

SNR RANGE $\gamma_{U}-\gamma_{L}$ FOR A NON-COHERENT FSK, NRZ RADIO capabilities of SECDED, which comes at a cost of energy efficiency (encoding ratio 1:3). NRZ, 4B5B, and Manchester does not provide error correction. Nevertheless, for the same packet size, the encoding ratios are 1:1, 1:1.25 and 1:2 respectively; resulting in higher SNRs required, which lead to smaller regions.

Though done for ASK modulation, empirical results for different encoding schemes [2] agree with the expected theoretical behavior, i.e. for the same environment, SECDED shows a larger connected region than Manchester encoding.

Even though the absolute radius of the regions are of interest in protocol evaluation, and their length can be obtained through equation 13; in the design of the radio, the main goal with regards to the regions- is to increase the connected region without increasing the transitional one. Henceforth, rather than comparing absolute distances, the $\Gamma$ coefficient will be used.

Table I shows the $\gamma_{U}-\gamma_{L}$ for the different encodings and frames size. The lower the $\gamma_{U}-\gamma_{L}$ the thinner the transitional region, SECDED shows the highest value among the encoding schemes; and, for a given encoding, as the frame size increases, $\gamma_{U}-\gamma_{L}$ decreases.

Finally, table II shows the $\Gamma$ coefficient of different radios in a environment with $n=4$ and $\sigma=4$. As we observe, the encoding or frame size do not have a significant impact on the

\begin{tabular}{|c|c|c|c|}
\hline encoding & 50 bytes & 100 bytes & 150 bytes \\
\hline NRZ & 1.8639 & 1.8240 & 1.8046 \\
4B5B & 1.8500 & 1.8130 & 1.7950 \\
Manchester & 1.8240 & 1.7923 & 1.7766 \\
SECDED & 1.9120 & 1.8540 & 1.8263 \\
\hline
\end{tabular}

TABLE II

$\Gamma$ COEFFICIENT FOR A NON-COHERENT FSK, NRZ RADIO 


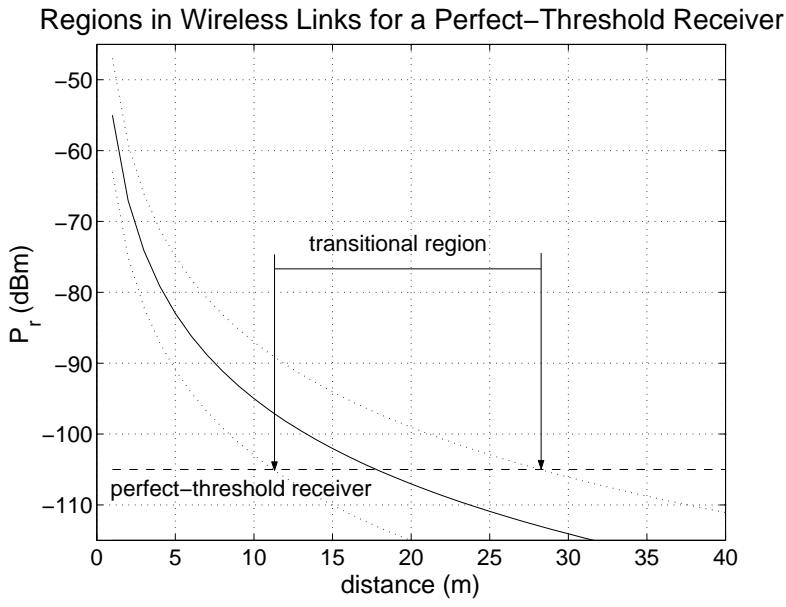

Fig. 8. Transitional Region for a Perfect-Threshold Receiver.

$\Gamma$ coefficient. No significant impacts were observed for other values of $n$ and $\sigma$ either.

\section{Can the transitional region be removed with a perfect- threshold radio?}

The previous section shows that modifying the encoding or packet size does not reduce significantly the $\Gamma$ coefficient, this result leads to an interesting question: Can the transitional region be removed with a perfect-threshold receiver (i.e. $\gamma_{U}=$ $\left.\gamma_{L}\right)$ ?. Figure 7 shows analytical PRR vs distance plots where perfect-threshold and real receivers are placed in ideal and real environments, by an ideal environment we refer to one with no shadowing effects $(\sigma=0)$; the real receiver and real environment follow the models derived in section III.

Figure 7 (a) shows the PRR vs distance for a perfectthreshold receiver in an ideal environment, this curve is the disc-shape model commonly used in many simulators. Figure 7 (b) shows a real receiver in an ideal environment, this scenario results in a small and deterministic transitional region, where the PRR decreases monotonically with respect to distance. Figure 7 (c) is the most interesting plot, it shows a perfectthreshold receiver in a real environment; in this scenario $\gamma_{U}=\gamma_{L}$, which leads to binary links ( 0 or 1$)$. Nevertheless, even in this ideal scenario the binary perfect-reception-withinrange model does not capture the behavior of the link, since there exist a region where a link can randomly take values of 0 or 1. Finally, figure 7d) shows the real behavior of the link.

Figure 8 shows the analytical behavior of a perfect-threshold receiver. We can observe that for a perfect-threshold receiver, the transitional region would be caused by the shadowing variance of the environment. Given that the shadowing variance is caused by multi-path effects, we hypothesize that, to have a significant impact in decreasing the transitional region, receivers should use mechanisms that combat multipath effects, such as spread spectrum and diversity techniques.

This subsection provides some important conclusions. First, a perfect-threshold receiver would not solve the transitional region problem due to multi-path effects. Second, in real scenarios, the radio is the cause of obtaining continuous values

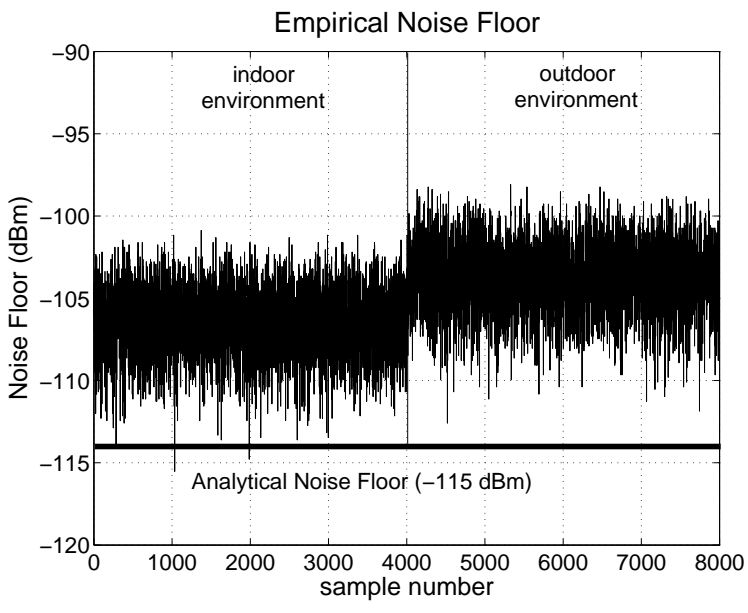

Fig. 9. Empirical Measurements of the Noise Floor.

of PRR, and not ' $0 / 1$ ' links; and the environment is the cause of the random (non-monotonically) decreasing trend. And third, given that multi-path effects play a significant role in determining the transitional region, receivers able to combat these effects may improve significantly the quality of the link.

\section{EMPIRICAL VAlidation OF THE Model}

In order to enhance and validate the theoretical model developed, MICA2 motes were used to perform empirical evaluation. First, we describe the methodology used. Then, we redefine the noise floor; after that, the channel parameters, $n$ and $\sigma$, are obtained; and the radio model is compared with the empirical results. Finally, we evaluate the accuracy of the analytical link layer model derived (PRR vs distance).

\section{A. Methodology}

Two different environments were tested, an indoor environment (aisle of a building), and an outdoor environment (football field). For each environment, a chain topology of 21 MICA2 motes was deployed with nodes spaced every meter. The frame size was 50 bytes and Manchester encoding was used with a preamble of 28 bytes. A simple TDMA protocol was implemented to avoid collisions. Upon reception of a packet the sequence number and the received signal strength $\left(P_{r}\right)$ were stored; simultaneously, the noise floor was measured by taking samples of the idle channel. For both environments; various power levels were tested (from $-20 \mathrm{dBm}$ to $5 \mathrm{dBm}$ in steps of $1 \mathrm{dBm})$, due to space constraints we present results for medium $(-7 \mathrm{dBm})$ and high $(5 \mathrm{dBm})$ powers. For each power level, each node transmitted 100 packets, at a rate of 5 packets/sec. After all nodes transmitted their 100 packets, the average $P_{r}$ (RSSI) and PRR were measured for all the links in the network.

\section{B. The Noise Floor}

Figure 9 shows samples of the noise floor for both environments. The average noise floor is approximately $-105 \mathrm{dBm}^{7}$,

\footnotetext{
${ }^{7}$ The noise floor difference in both environments is due to slightly different temperatures, and sensitivity inaccuracies $( \pm 6 \mathrm{~dB}[14])$.
} 


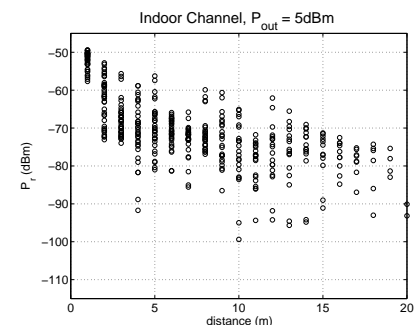

(a)

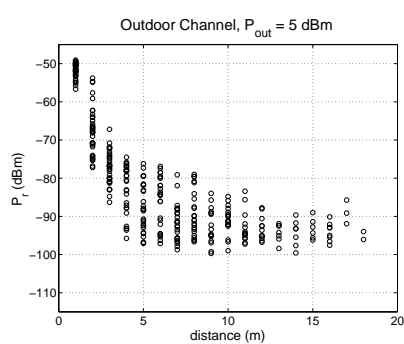

(b)

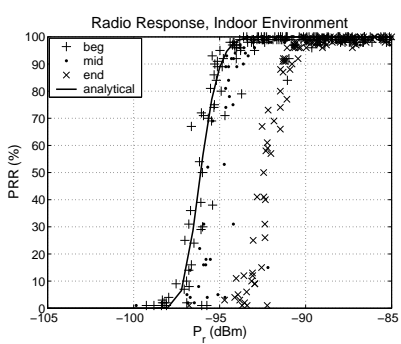

$(c)$

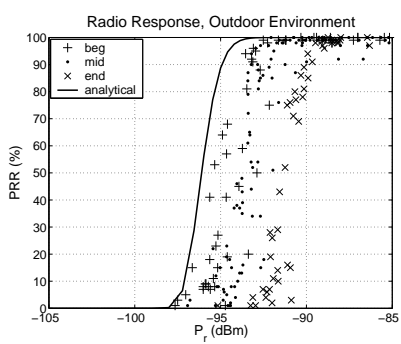

$(d)$

Fig. 10. Empirical Validation of Channel and Radio Models. (a) Indoor Channel (b) Outdoor Channel (c) Indoor Radios (d) Outdoor Radios

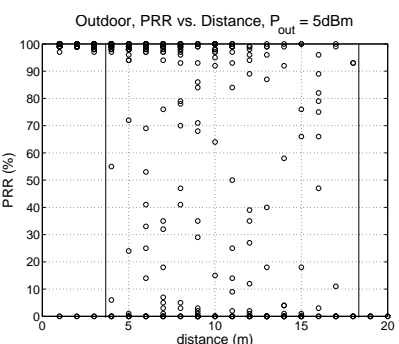

(a)

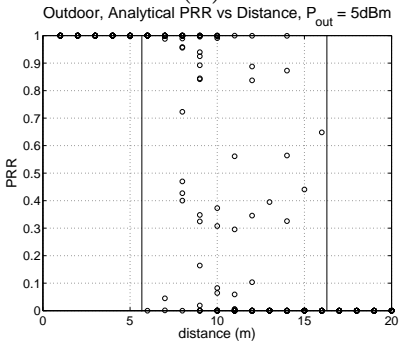

(c)

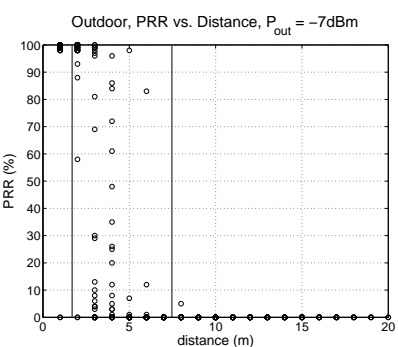

(b)

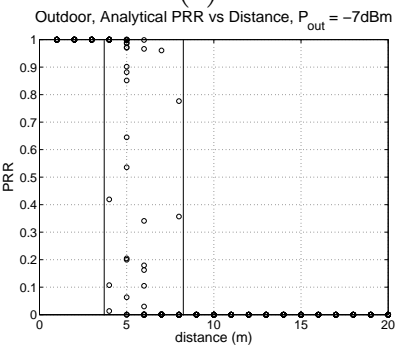

$(d)$
Fig. 11. Validation of the Model for Outdoor Channel. (a) and (b) are the empirical measurements; (c) and (d) the analytical counterparts

which has a $10 \mathrm{dBm}$ difference with respect to the specified value obtained in section III-C. This difference is mainly due to the fact that in the initial calculation we did not consider the losses from the output of the chip to the antenna. These losses depend on the board implementation and are beyond the scope of this work. Hence, for the model, let us redefine the noise floor to an average value of $-105 \mathrm{dBm}$.

\section{The Channel and Radio Models}

The channel parameters ( $n$ and $\sigma$ ) were obtained from figures 10 (a) and 10 (b). However, there was a small complication to obtain them in the outdoor environment - figure 10 (b). Due to the noise floor, $P_{r}$ values below $-100 \mathrm{dBm}$ were not detected (values were recorded only for received packets). For this reason, only the closest distances $(1 \mathrm{~m} \sim 6 \mathrm{~m})$ were considered in the curve-fitting. Table III shows the parameters for both environments.

Figures 10 (c) and 10 (d) show the radio model and the PRR vs $P_{r}$ of three different receivers for both environments; the receivers were located at the beginning, middle and end of the chain. The radio model was obtained from the parameters used in the deployment. Since the model is based on SNRs, it

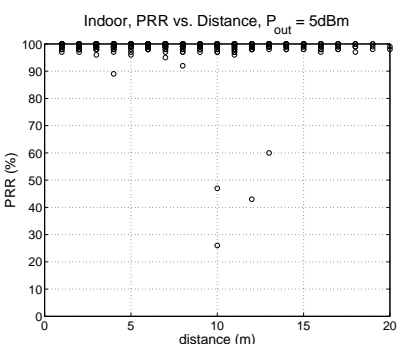

(a)

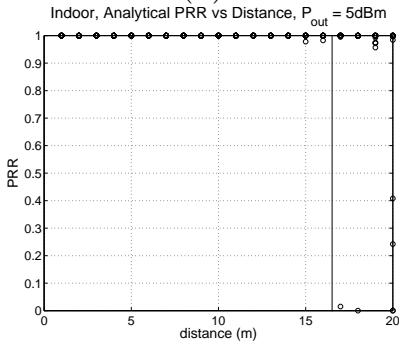

$(c)$

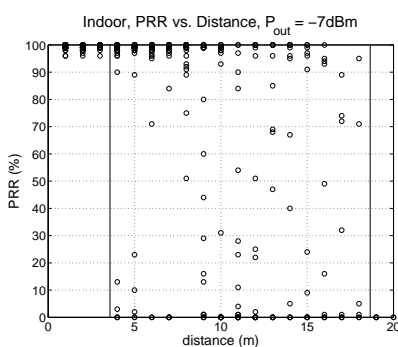

(b)

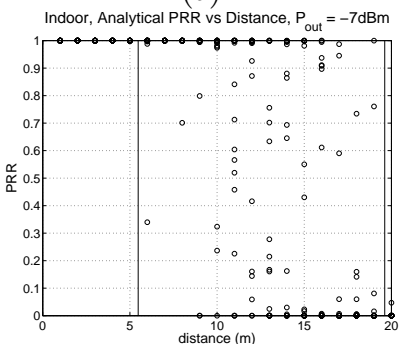

$(d)$
Fig. 12. Validation of the Model for Indoor Channel. (a) and (b) are the empirical measurements; (c) and (d) the analytical counterparts

was shifted according to the average noise floor $(-105 \mathrm{dBm})$. An analogous trend is observed on the empirical response of the receivers and the radio model; furthermore, the radio response is independent of the environment due to the similar temperatures in both scenarios.

\section{The Link Layer Model}

For the parameters used in the experiments, table IV shows the expected radius of the different regions. Figures 11 and 12 show the empirical and analytical results of the link layer abstraction (PRR vs distance) for the outdoor and indoor environments, respectively.

Figures 11 (a), 11 (b), 12 (a) and 12 (b) correspond to the empirical results; and figures 11 (c), 11 (d), 12 (c) and 12 (d) are their analytical counterparts. The radius obtained in

\begin{tabular}{|l|c|c|}
\hline environment & $n(95 \%$ conf. bounds) & $\sigma(95 \%$ conf. bounds $)$ \\
\hline outdoor & $4.7(4.30-5.10)$ & $4.6(2.80-6.40)$ \\
indoor & $3.0(2.67-3.23)$ & $3.8(2.60-5.00)$ \\
\hline
\end{tabular}

TABLE III

Channel Parameters 


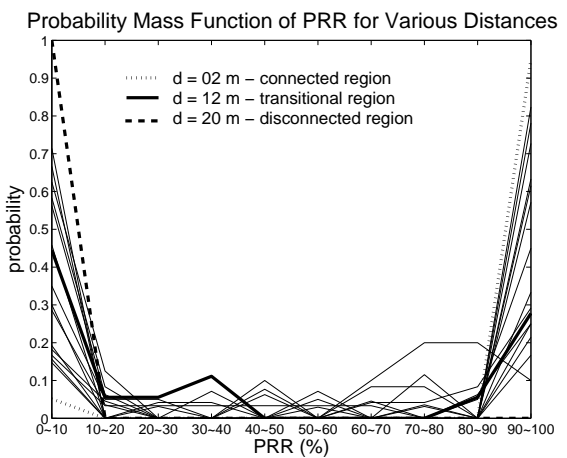

(a)

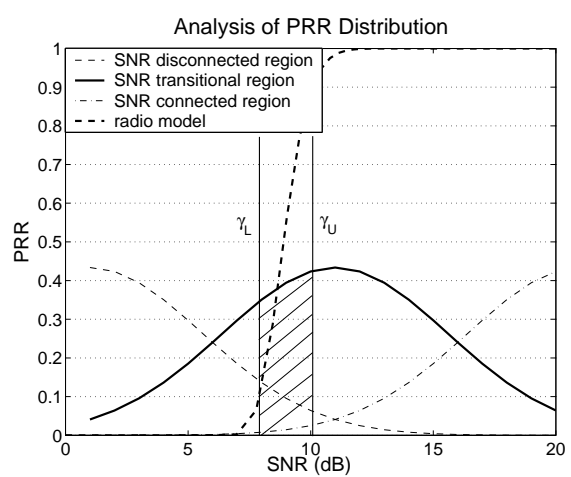

$(b)$

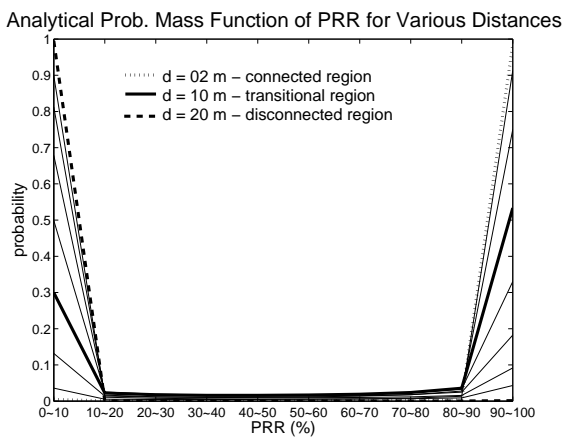

$(c)$

Fig. 13. PRR Distribution for Different Transmitter-Receiver Distances. (a) empirical distribution of the high-power-outdoor environment, (b) analytical observation of the distribution, (c) analytical distribution of the high-power-outdoor environment

table IV fairly approximate the real behavior. Also, by simple inspection a similar distribution of the PRRs is observed. However, in order to verify the correctness of the model, let us compare the empirical and analytical distributions of the PRR as a function of the transmitter-receiver distance. For example, a link in the connected region is expected to have, with high probability, a PRR above $90 \%$; while a link in the transitional region, depending on whether the node is at the beginning, middle or end of this region, will have different PRR distributions. Notice that the distributions depend on the transmitter-receiver distance for the given channel and radio parameters, and are constant in time due to the focus on static environments of this work.

In figure 13 (a), each curve shows the PRR distribution of transmitter-receiver distances between 2 and $20 \mathrm{~m}$ (in steps of $1 \mathrm{~m}$ ) for the outdoor-high-power scenario. Three curves are specially highlighted, one on each region. As expected, curves in the connected and disconnected regions show a high probability $(\sim 1)$ of having high $(90 \%<)$ and low $(<10 \%)$ PRRs, respectively. However, the curve in the transitional region -all curves in general- show a strong bias to either high or low PRRs, with a small probability of being between $10 \%$ and $90 \%$. This behavior can be explained in light of the model derived in this work.

Figure 13 (b) shows the radio model and three SNR distributions of tentative receivers. The left-most curve represents a node in the disconnected region, where low SNR values result in low PRRs. The right-most curve represents a node in the connected region, which contrary to the previous curve results in high PRRs. And, the middle curve represents a node in the transitional region.

For the curve in the transitional region, the probability that

\begin{tabular}{|l|c|c|}
\hline scenario & $d_{s}(\mathrm{~m})$ & $d_{e}(\mathrm{~m})$ \\
\hline outdoor-high-power & 5.7 & 15.5 \\
outdoor-medium-power & 3.2 & 8.6 \\
indoor-high-power & 17.4 & 65.1 \\
indoor-medium-power & 6.9 & 25.9 \\
\hline
\end{tabular}

TABLE IV

ANALytical Radius of Transitional REgion an SNR value falls within the $\gamma_{U}-\gamma_{L}$ region is low compared to the probability of falling either in the high or low regions. It is important to remark -and can be easily observed- that independently of where the SNR distribution is centered, SNR values have a low probability of falling within the $\gamma_{U}-\gamma_{L}$ region. Figure 13 (c) is the analytical counterpart of figure 13 (a) for the high-power-outdoor environment. Similar trends are obtained for other scenarios.

Finally, one of the goals of this work is to provide a realistic link layer model for low power devices. With that aim, table $\mathrm{V}$ presents a comprehensive list of equations for different modulation and encoding techniques ${ }^{8}$.

\section{CONCLUSions AND Future WORK}

The impact that the channel behavior has on the performance of upper-layer protocols in wireless sensor networks requires a clear understanding of the different regions of low power wireless links. We have presented a detailed study of the transitional region. Some of the key contributions and conclusions of this work are:

- Mathematical link layer models are presented for the statistical variation of packet reception rates with respect to distance (for different environment and radio characteristics). This analysis yields the boundaries of the different regions - connected, transitional, and disconnected. The methodology presented can be easily extended to other radios that use different modulation and encoding schemes.

- The study shows the influence that the modulation, encoding, output power, frame size, noise floor, and channel parameters have on the transitional region.

- The $\Gamma$ (transitional region) coefficient is introduced as a means to compare the quality of the link for different environments. The smaller the coefficient, the better the link. Environments with a high path loss exponent $n$ and a small shadowing standard deviation $\sigma$, decrease the $\Gamma$ coefficient. Also, while the frame size and encoding scheme influences the radius of the regions, their ratio $(\Gamma$ coefficient) is not significantly affected.

\footnotetext{
${ }^{8}$ The model assumes that the preamble is not encoded, and hence is the same for all encoding schemes. Other radio designs may lead to slightly different expressions.
} 


\begin{tabular}{|c|c|}
\hline STEP 1: Channel & Obtain parameters of the channel and use them in next step \\
\hline$P L\left(d_{0}\right), n, \sigma$ & Can be obtained through own empirical measurements, or from some published results [10] \\
\hline STEP 2 : SNR & Obtain SNR $\gamma$ as a function of distance $d$. For MICA2: $-20 d B m<P_{t}<5 d B m, P_{n}=-105 d B m$ \\
\hline$\gamma_{d B}(d)$ & $P_{t}-P L\left(d_{0}\right)-10 n \log _{10}\left(\frac{d}{d_{0}}\right)-N(0, \sigma)-P_{n}$ \\
\hline STEP 3 : Modulation & $\begin{array}{l}\text { Choose } P_{e} \text { according to the modulation used, insert } \gamma(d) \text { not in } \mathrm{dB} \text {, i.e. } 10^{\frac{\gamma_{d B}(d)}{10}} \text {, and convert from } \frac{E_{b}}{N_{0}} \text { to } R S S I \\
\text { by inserting the appropriate bit data rate } R \text { and noise bandwidth } B_{N}\end{array}$ \\
\hline ASK & noncoherent: $\frac{1}{2}\left[\exp ^{-\frac{\gamma(d)}{2}} \frac{B_{N}}{R}+Q\left(\sqrt{\gamma(d) \frac{B_{N}}{R}}\right)\right] \quad$ coherent: $Q\left(\sqrt{\frac{\gamma(d)}{2} \frac{B_{N}}{R}}\right)$ \\
\hline FSK & noncoherent: $\frac{1}{2} \exp ^{-\frac{\gamma(d)}{2}} \frac{B_{N}}{R}$ \\
\hline PSK & binary: $Q\left(\sqrt{2 \gamma(d)} \frac{B_{N}}{R}\right)$ \\
\hline STEP 4 : Encoding & Choose packet reception rate $p(d)$ according to the encoding scheme, frame and preamble lengths \\
\hline $\begin{array}{l}\text { NRZ } \\
\text { 4B5B } \\
\text { Manchester } \\
\text { SECDED }\end{array}$ & $\begin{array}{l}\left(1-P_{e}\right)^{8 \ell}\left(1-P_{e}\right)^{8(f-\ell)} \\
\left(1-P_{e}\right)^{8 \ell}\left(1-P_{e}\right)^{8(f-\ell) 1.25} \\
\left(1-P_{e}\right)^{8 \ell}\left(1-P_{e}\right)^{8(f-\ell) 2.0} \\
\left(1-P_{e}\right)^{8 \ell}\left(\left(1-P_{e}\right)^{8}+8 P_{e}\left(1-P_{e}\right)^{7}\right)^{(f-\ell) 3.0}\end{array}$ \\
\hline
\end{tabular}

TABLE V

THEORETICAL MODELS FOR THE LINK LAYER

- Even with a perfect-threshold radio, the transitional region still exists so long as there are multi-path effects. However, we hypothesize that radios with mechanisms to combat multi-path effects, such as spread-spectrum and diversity techniques, can reduce the transitional region.

Even though interference was not studied, the channel model can be used to consider $P_{r}$ signals of non-intended receivers as noise. For scenarios where the traffic and contention are relatively light; a very reasonable assumption for many classes of data-centric sensor networks, the presented model provides an accurate estimate of the links' quality. However, a more detailed study is needed to accurately quantify the impact of interference in the different regions for high-traffic networks.

Our work focused on the spatial variation of the link and we did not consider the time domain. We believe that in static environments (i.e. static nodes, and no dynamic objects around them) time variations are mainly due to fluctuations in the thermal noise of the radios. Then, the time variations could be modelled by a gaussian distribution of the thermal noise, and use these samples as the noise floor for each packet, instead of the deterministic value assumed in this work. For more challenging dynamic environments, richer time-varying fading models will be required; for example, developing good models for the correlated temporal variations of the $X_{\sigma}$ term in the log-normal shadowing model. We would also like to extend our modelling and analysis to more sophisticated radios that implement techniques such as spread spectrum and multiantenna diversity to combat fading effects.

\section{ACKNOWLEDGEMENT}

We would like to acknowledge assistance from and useful conversations with Alec Woo, Dr. Jerry Zhao, Prof. John Heidemann, Dr. Robert Poor, and Prof. Scott Shenker.

\section{REFERENCES}

[1] D. Ganesan, B. Krishnamachari, A. Woo, D. Culler, D. Estrin and S. Wicker."Complex Behavior at Scale: An Experimental Study of
Low-Power Wireless Sensor Networks". UCLA CS Technical Report UCLA/CSD-TR 02-0013, 2002.

[2] J. Zhao and R. Govindan. "Understanding Packet Delivery Performance in Dense Wireless Sensor Networks". Sensys '03.

[3] A. Woo, T. Tong, and D. Culler. "Taming the Underlying Issues for Reliable Multhop Routing in Sensor Networks". SenSys '03.

[4] A. Cerpa, N. Busek, and D. Estrin. "SCALE: A tool for Simple Connectivity Assessment in Lossy Environments". CENS Technical Report, September 2003.

[5] D. S. J. De Couto, D. Aguayo, J. Bicket, and R. Morris. "A HighThroughput Path Metric for Multi-Hop Wireless Routing". ACM MobiCom, September 2003.

[6] D. Kotz, C. Newport and C. Elliott. "The mistaken axioms of wirelessnetwork research". Technical Report TR2003-467, Dept. of Computer Science, Dartmouth College, July 2003.

[7] G. Zhou, T. He, S. Krishnamurthy, and J. Stankovic. "Im- pact of radio irregularity on wireless sensor networks". MobiSys '04.

[8] A. Cerpa, J. L. Wong, L. Kuang, M. Potkonjak and D. Estrin. "Statistical Model of Lossy Links in Wireless Sensor Networks". CENS Technical Report 0041, April 2004.

[9] D. Lal, A. Manjeshwar, F. Herrmann, E. Uysal-Biyikoglu, A. Keshavarzian. "Measurement and Characterization of Link Quality Metrics in Energy Constrained Wireless Sensor Networks". Globecom 2003.

[10] K. Sohrabi, B. Manriquez, and G. Pottie. "Near Ground Wideband Channel Measurement". Vehicular Technology Conference IEEE, volume 1, pages 571-574, 1999.

[11] S. Y. Seidel and T. S. Rapport. "914 MHz Path Loss Prediction Model for Indoor Wireless Communication in Multi floored Buildings". In IEEE Transactions on Antennas and Propagation, volume 40(2), pages 207217, February 1992.

[12] H. Nikookar and H. Hashemi. "Statistical modeling of signal amplitude fading of indoor radio propagation channels". 2nd International Conference on Universal Personal Communications, 1993. Vol 1, Pages:84-88.

[13] Theodore S. Rappapport. "Wireless Communications: Principles and Practice". Prentice Hall.

[14] Chipcon. CC1000 low power radio transceiver, http://www.chipcon.com. 